\title{
Study of the leakage field of magnetic force microscopy thin-film tips using electron holography
}

\author{
B. G. Frost \\ EM Facility, University of Tennessee, 1414 West Cumberland Avenue, Knoxville, Tennessee 37995-0810 \\ N. F. van Hulst \\ Applied Optics Group, Department of Applied Physics, University of Twente, P.O. Box 217, \\ 7500 AE Enschede, The Netherlands \\ E. Lunedei and G. Matteucci \\ Department of Physics, University of Bologna, V/le B. Pichat 6, 40126 Bologna, Italy \\ E. Rikkers \\ Transducers and Materials Science Group, Department of Electrical Engineering, University of Twente, \\ P. O. Box 217, 7500 AE Enschede, The Netherlands
}

(Received 12 June 1995; accepted for publication 24 January 1996)

\begin{abstract}
Electron holography is applied for the study of the leakage field of thin-film ferromagnetic tips used as probes in magnetic force microscopy. We used commercially available pyramidal tips covered on one face with a thin NiCo film, which were then placed in a high external magnetic field directed along the pyramid axis. Good agreement between simulated and experimental electron phase difference maps allows to measure the local flux from the ferromagnetic tips and therefore to evaluate the perturbation induced by the microprobe stray field on the sample area. (C) 1996 American Institute of Physics. [S0003-6951(96)03813-5]
\end{abstract}

Magnetic force microscopy (for a review see Ref. 1 and references therein) is one specific technique, which permits the imaging of near-surface magnetic microfields at sub100-nm lateral resolution and the determination of magnetic properties of thin films on nanometer scale. ${ }^{2}$ In this form of microscopy a sample is raster scanned by a probe and the physical interaction is monitored.

The magnetic field distribution around the tip apex must be known exactly in order to be able to interpret the acquired image with respect to quantitative magnetization determination.

Off axis electron holography ${ }^{3}$ was used for revealing the magnetic field around the tip apex. This method enables a direct display of the magnetic field lines of force surrounding the tip., ${ }^{4,5}$

The magnetic sensor in this study is based on commercially available silicon-nitride atomic force microscopy tips positioned at the end of a cantilever. The pyramidal tip is subsequently coated with a $100 \mathrm{~nm}$ layer of magnetic material by evaporation, under a small angle $\alpha$, of ferromagnetic $\mathrm{Co}_{80} \mathrm{Ni}_{20}$ according to Fig. 1. The magnetic material covers only one of the four tip faces. After the deposition of the magnetic material, the tip is poled in the homogeneous part of a $1 T$ field which is oriented along the tip axis, perpendicular to the cantilever.

The electron holograms were recovered by using an electrostatic biprism as an interferometry device, as sketched in Fig. 2. By applying a suitable voltage to the wire, the part of the wave illuminating the sample (object wave, left-hand side of the fiber) is brought to interfere with a part of the wave which travels a few microns distant (reference wave). The latter is also modulated by the leakage field of the tip. Therefore in the holograms, the phase difference between the object and the reference waves will be recorded.
A Hitachi HF-2000 field emission electron microscope operating at $200 \mathrm{kV}$ with a Möllenstedt biprism inserted between the first and second intermediate lenses was used to investigate the probe.

The objective lens was switched off to exclude any interaction between the magnetic lens field and the sample. In addition, the probe was coated with a thin gold layer to minimize electric charging by the electron beam.

Our holograms are acquired by a 1024 by 1024 pixel slow-scan CCD-camera and processed by the software "HoloWorks" (Gatan Inc. $\left.{ }^{6}\right)$. This software facilitates, among other things, automated reconstruction of the image phase.

Figure 3 reports the experimental phase map due to the leakage field around the tip. The very white regions (or alternatively the very black ones) can be directly interpreted as magnetic lines of force. The projected image of the tip can be easily recognized within the interference field region whose width is about $5 \mu \mathrm{m}$.

The magnetic layer deposited on the tip is shown by the bright line on the left-hand side. The pattern can be divided into three regions: (i) left-hand side: starting from the mag-

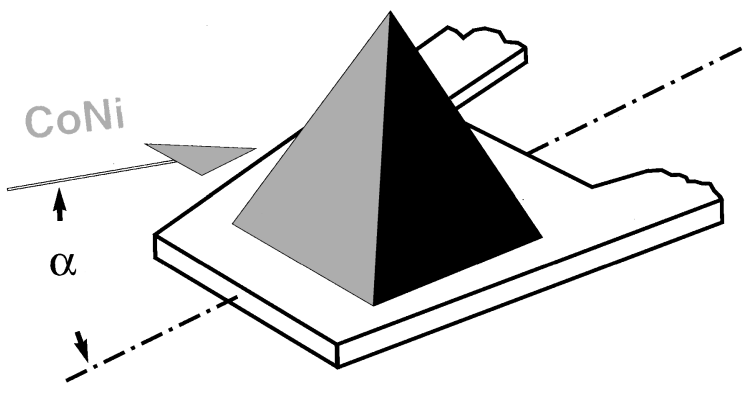

FIG. 1. Schematic drawing of the coating procedure of the tip. The angle $\alpha$ is positive and only a few degrees. 


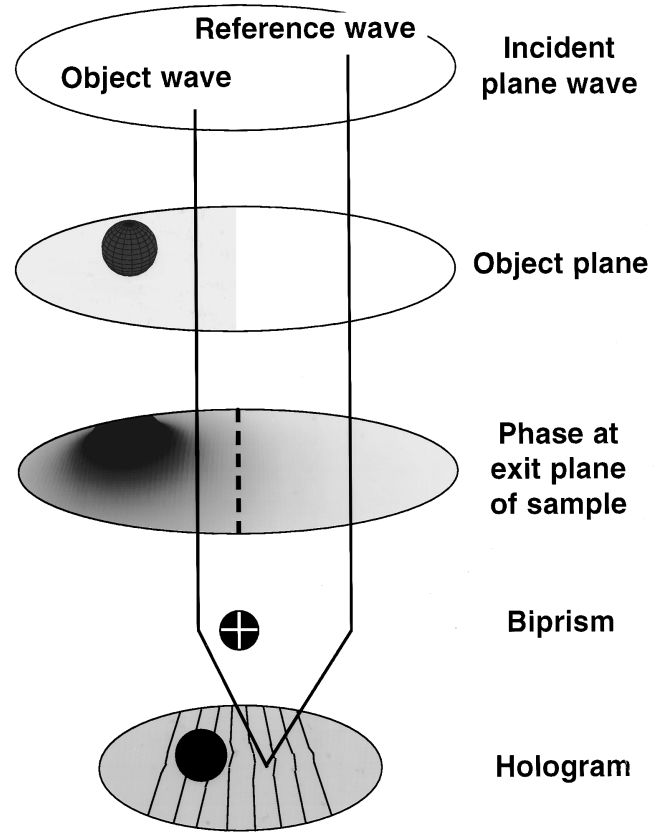

FIG. 2. Schematic arrangement for image hologram formation.

netic layer, the lines assume a rounded shape and then join the same face near the pyramid base; (ii) tip apex; only one line is shown which crosses the whole interference region along the tip axis direction; (iii) right-hand side, the flux closure is displayed on one face of the tip not coated with the NiCo film.

The magnetic field leaking from the apex of the pyramidal probe has been simulated by assuming that the magnetic domain structure of the layer could be replaced by a uniform distribution of magnetic dipoles.

The thin magnetic film area was divided into a number of regions whose average magnetic moment is oriented along the axis of the associated magnetic dipole. Polar coordinates were used for the description of the angular configuration of each dipole. Such a configuration is then minimized in its total free energy by means of ad hoc developed programs.

Two main terms were taken into account for energy balance: ${ }^{7}$ the energy of dipole-dipole interaction and the energy associated to the interaction between the magnetic moment of each single dipole and the external applied magnetic field. The limit angular configuration of all dipoles is such that each dipole is oriented parallel to the vectorial sum of

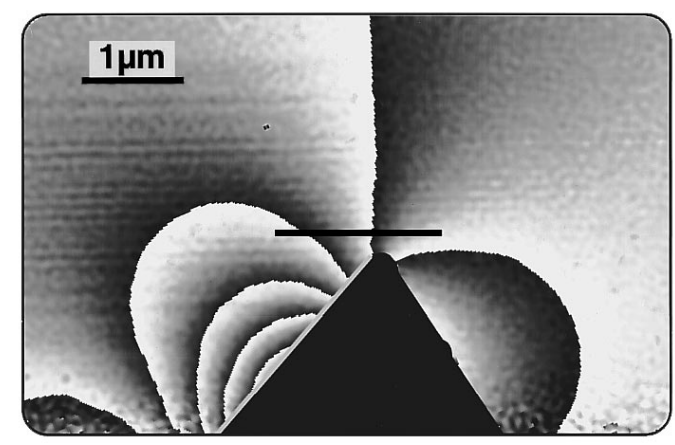

FIG. 3. Magnetic lines of force around the pyramidal tip.

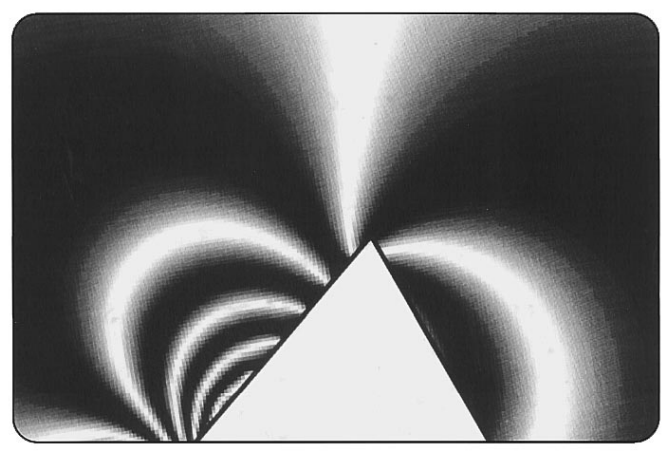

FIG. 4. Simulation of the same region as Fig. 3.

the external applied magnetic field and the global magnetic field generated by all other dipoles.

Each dipole can rotate freely around the polar angles, but no dipole shift is allowed. This magnetic system evolves from a completely random angular distribution of the dipoles by adjusting the angles of all dipoles until it reaches a local minimum energy condition. By repeating this process many times and tabulating the angular configurations reached by the system we can evaluate the final arrangements that most likely are obtained. This approach, although simplified, has the merit of providing a good agreement between the experimental results and the simulations.

In order to map the leakage field around the tip, the phase shift on the electron wave due to the presence of the set of dipoles, in their final angular configuration, can be calculated according to the Aharonov-Bohm effect and using the Stokes theorem. ${ }^{4,5}$

The resulting pattern is shown in Fig. 4 where the same interference field of Fig. 3 is displayed. A close comparison between the experimental result and the simulated map shows the validity of the proposed model for the magnetic thin-film tip and in particular for the description of the field leaking out from the apex.

Possible variations of the phase distribution on the final map due to a reference wave modulated by the leakage field of the tip have been duly considered. As the reference wave is very weakly affected, simulations show that no significant changes are produced on the contour map.

An estimate of the total flux in the region of interest, around the tip apex, can be made through the evaluation of the total phase difference. A horizontal line close to the tip apex is drawn to indicate the surface of a sample at a typical working distance in a magnetic force microscope. The total flux affecting the sample can be determined by counting the number of black lines since between two successive ones a magnetic flux $h / e$ is enclosed.

The magnetic field produced by thin-film microprobes has been quantitatively analyzed by electron holography. These results are important in the interpretation of the experimental observations in magnetic force microscopy. Further work is in progress to investigate the interaction of the probe field with the sample under investigation.

The experimental work was performed at the Oak Ridge National Laboratory, sponsored by the Laboratory Directed R\&D Program of Oak Ridge National Laboratory, managed for the DOE by Lockheed Martin Energy Research, under 
Contract DE-AC05-84OR21400. This work was financially supported by MURST-Italy. We thank L. Allard and E. Völkl for helpful discussions.

${ }^{1}$ U. Hartmann, Adv. Electron. Electron Phys. 87, 49 (1994).

${ }^{2}$ H. teLintelo, Ph.D. thesis, University of Twente, Enschede, Holland, 1993.
${ }^{3}$ A. Tonomura, Adv. Phys. 41, 59 (1992).

${ }^{4}$ G. Matteucci, M. Muccini, and U. Hartmann, Appl. Phys. Lett. 62, 1839 (1993).

${ }^{5}$ G. Matteucci, M. Muccini, and U. Hartmann, Phys. Rev. B 50, 6823 (1994).

${ }^{6}$ E. Völkl, L. F. Allard, and B. Frost, J. Microscopy 180, 39 (1995).

${ }^{7}$ A. H. Morrish, The Physical Principles of Magnetism (Robert E. Krieger, Malabar, FL, 1983), p. 337. 